\title{
On 2-absorbing commutative semigroups and their applications to rings
}

\author{
A. Yousefian Darani • E.R. Puczyłowski
}

Received: 2 August 2011 / Accepted: 26 June 2012 / Published online: 13 July 2012

(C) The Author(s) 2012. This article is published with open access at Springerlink.com

\begin{abstract}
A commutative ring $R$ is called 2-absorbing (Badawi in Bull. Aust. Math. Soc. 75:417-429, 2007) if for arbitrary elements $a, b, c \in R, a b c=0$ if and only if $a b=0$ or $b c=0$ or $a c=0$. In this paper we study this concept in a more general framework of commutative (multiplicative) semigroups with 0 . The results obtained apply to many ring theoretic situations and make it possible to describe similarities and differences among some variants of the notion. We pay a particular attention to graded rings. We also show that a conjecture from (Anderson and Badawi in Commun. Algebra 39:1646-1672, 2011) concerning $n$-absorbing rings holds for rings with torsion-free additive groups.
\end{abstract}

Keywords Commutative rings $\cdot$ Commutative semigroups $\cdot 2$-Absorbing rings Prime ideals

\section{Introduction and preliminaries}

A commutative ring $R$ is called 2-absorbing (cf. [2]) if for arbitrary elements $r_{1}, r_{2}, r_{3}$ of $R$ such that $r_{1} r_{2} r_{3}=0$ there are $1 \leq i \neq j \leq 3$ for which $r_{i} r_{j}=0$. Obviously 2 -absorbing rings generalize prime rings. In [2] the structure of such rings was described and it was applied to show that a ring $R$ is 2 -absorbing if and only if for

Communicated by László Márki.

E.R. Puczyłowski was supported by Polish MNiSW grant No. N N201 268435.

A. Yousefian Darani

Department of Mathematics, University of Mohaghegh Ardabili, P.O. Box 179, Ardabil, Iran e-mail: yousefian@uma.ac.ir

E.R. Puczyłowski ( $₫)$

Institute of Mathematics, University of Warsaw, Banacha 2, 02-097 Warsaw, Poland

e-mail: edmundp@mimuw.edu.pl 
arbitrary ideals $I_{1}, I_{2}, I_{3}$ of $R$ such that $I_{1} I_{2} I_{3}=0$ there are $1 \leq i \neq j \leq 3$ for which $I_{i} I_{j}=0$. Thus 2-absorbing rings can be defined in two equivalent ways, by elements or by ideals. It was quite intriguing for us that the proof of this result was rather non-trivial, whereas the respective characterization of prime rings by elements or by ideals is immediate. It became even more interesting when we observed that for graded rings the respective notions defined in terms of homogeneous elements and homogeneous ideals are already not equivalent. Trying to explain that phenomena we came to a conclusion that it is more appropriate for such studies to take the context of commutative (multiplicative) semigroups with 0 . The definition of 2-absorbing rings as well as their ideal characterization are given in terms of the multiplication only, so they extend immediately to commutative semigroups with 0 . One my expect that the results valid for rings extend to that more general situation. However it is not hard to construct examples showing that in this case the concepts defined by elements and ideals are already not equivalent. More important is that semigroups defined by the counterpart of the condition involving ideals can indeed be described similarly to 2-absorbing rings and the results obtained in this way can be applied to many ring theoretic situations, say, to all rings (giving the results obtained in [2]) or graded rings (which we do in this paper). This approach helped us also to specify the role of the additive structure of rings (or even semirings) in establishing that for rings the concepts defined by elements and ideals are equivalent.

One can define in an obvious way the concepts of $n$-absorbing rings for integers $n \geq 2$. It seems however that it might be hard to extend the results known for $n=2$ to higher integers. Some studies concerning that more general situation were recently started in [1] where it was conjectured that also in this case the notions defined by elements and ideals coincide. We show that this conjecture holds for rings whose additive groups are torsion-free. Our arguments indicate also one of the reasons for which the cases $n=2$ and $n \geq 3$ are substantially different.

Throughout the paper $S$ denotes a commutative semigroup with 0 .

We say that $S$ is 2-absorbing if for arbitrary elements $s_{1}, s_{2}, s_{3} \in S$ satisfying $s_{1} s_{2} s_{3}=0$ there are $1 \leq i \neq j \leq 3$ such that $s_{i} s_{j}=0$ and we say that $S$ is strongly 2-absorbing if for arbitrary ideals $I_{1}, I_{2}, I_{3}$ of $S$ satisfying $I_{1} I_{2} I_{3}=0$ there are $1 \leq$ $i \neq j \leq 3$ such that $I_{i} I_{j}=0$.

It is clear that strongly 2-absorbing semigroups are 2-absorbing. The following simple example shows that the converse does not hold.

Example 1.1 Let $S$ be the Rees factor of the free commutative semigroup generated by $x, y$ modulo the ideal $I$ generated by $x^{2}, y^{2}$. It is easy to see that $S^{3}=0$ but $S^{2} \neq 0$. Hence $S$ is not strongly 2-absorbing. If $w_{1}, w_{2}, w_{3}$ are arbitrary elements of the free semigroup, then two of them, say $w_{1}, w_{2}$, both contain $x$ or both contain $y$. Hence $w_{1} w_{2} \in I$, which shows that $S$ is 2-absorbing.

We start our studies with describing the structure of strongly 2-absorbing semigroups and next apply the obtained results to rings and rings graded by abelian groups. It is clear that they can be applied to many other ring (or even semiring) situations. We show also that the multiplicative semigroups of commutative rings are 2 -absorbing if and only they are strongly 2-absorbing. In the last section we show 
that similar arguments can be applied to verify the above mentioned conjecture from [1] for rings whose additive groups are torsion-free.

\section{Characterizations of strongly 2-absorbing semigroups}

In what follows $B$ denotes the prime radical of $S$, i.e., the intersection of all prime ideals of $S$. Since $S$ is commutative, $B$ coincides with the set of all nilpotent elements of $S$. For arbitrary elements $x, y \in S$ we denote by $(x)$ and $(x, y)$ the ideals of $S$ generated by $x$ and $x, y$, respectively.

Proposition 2.1 If $S$ is strongly 2-absorbing, then $B^{2}=0$.

Proof For every $s \in B$ there is a positive integer $n$ such that $(s)^{n}=0$. Applying the assumption that $S$ is strongly 2-absorbing one easily gets $(s)^{2}=0$, so $s^{2}=0$. This implies that for arbitrary $s, t \in B,(s)(s, t)(t)=0$. Hence since $S$ is strongly 2-absorbing, $(s)(t)=0$ or $(s)(s, t)=0$ or $(s, t)(t)=0$. In all cases $s t=0$. Hence $B^{2}=0$.

Example 1.1 shows that there are nilpotent 2-absorbing semigroups which are not strongly 2-absorbing. There are also semiprime semigroups of that type.

Example 2.2 Let $S$ be the Rees factor of the free commutative semigroup $W$ generated by $x, y, z$ modulo the ideal $I$ generated by $x y, y z, z x$. It is evident that $S$ contains no non-zero nilpotent element. If $w_{1}, w_{2}, w_{3} \in W$ and $w_{1} w_{2} w_{3} \in I$, then one of the words $x y, y z, z x$ must be a subword of $w_{i} w_{j}$ for some $1 \leq i \neq j \leq 3$, so $w_{i} w_{j} \in I$. Hence $S$ is 2-absorbing. Now $(x, y)(y, z)(z, x) \subseteq I$ but no product of the two ideals $(x, y),(y, z),(z, x)$ is contained in $I$, so $S$ is not strongly 2-absorbing.

The following characterization of strongly 2-absorbing semigroups is quite useful as it allows one to apply induction arguments in some investigations.

Proposition 2.3 $S$ is strongly 2-absorbing if and only if for arbitrary ideals $F_{1}, F_{2}, F_{3}$ generated by $\leq 2$ elements each and satisfying $F_{1} F_{2} F_{3}=0$ there are $1 \leq i \neq j \leq 3$ such that $F_{i} F_{j}=0$.

Proof Let $X, Y, Z$ be ideals of $S$ such that $X Y Z=0$. Suppose that $X Y \neq 0, Y Z \neq$ $0, X Z \neq 0$. Then there are $x_{1}, x_{2} \in X, y_{1}, y_{2} \in Y, z_{1}, z_{2} \in Z$ such that $x_{1} y_{1} \neq$ $0, x_{2} z_{1} \neq 0, y_{2} z_{2} \neq 0$. Let $F_{1}=\left(x_{1}, x_{2}\right), \quad F_{2}=\left(y_{1}, y_{2}\right), F_{3}=\left(z_{1}, z_{2}\right)$. Clearly $F_{1} F_{2} F_{3}=0$ but $F_{i} F_{j} \neq 0$ for arbitrary $1 \leq i \neq j \leq 3$. This proves the "if" part. The "only if" part is clear.

For a given subset $X$ of $S$ we denote by $\operatorname{ann}(X)$ the annihilator of $X$ in $S$, i.e., $\operatorname{ann}(X)=\{s \in S \mid X s=0\}$.

Now we pass to study the structure of strongly 2-absorbing semigroups.

Theorem 2.4 S is strongly 2-absorbing if and only if 
(a) If $I, J$ are ideals of $S$ such that $I \nsubseteq B, J \nsubseteq B$ and $I J \subseteq B$, then $I J=0$ and $I B=0=J B$

(b) For every subset $X$ of $B$, ann $(X)$ is a prime ideal of $S$;

(c) If $I, J, K$ are ideals of $S$ not contained in $B$, then $I J \neq 0$ or $J K \neq 0$ or $I K \neq 0$.

Proof Suppose that $S$ is strongly 2-absorbing.

(a) By Proposition $2.1, B^{2}=0$. Hence since $I J \subseteq B$, we have $B I J=0$. Suppose that $I J \neq 0$. Then $B I=0$ or $B J=0$. If, say, $B I=0$, then $I^{2} J=0$. However $I \nsubseteq B$, so $I^{2} \neq 0$. Since also $I J \neq 0$, we get a contradiction with the assumption that $S$ is strongly 2-absorbing. Hence $I J=0$. Now $(B \cup I) J \subseteq B$, so by the foregoing $(B \cup I) J=0$ and further $B J=0$. Similarly $B I=0$.

(b) Suppose that $I, J$ are ideals of $S$ such that $X I J=0$. We can assume that $X$ is an ideal of $R$. If $I \subseteq B$ or $J \subseteq B$, then since $B^{2}=0$, we get that $X I=0$ or $X J=0$. Assume that both $I, J$ are not contained in $B$. If $I J \neq 0$, then $X I=0$ or $X J=0$ as $S$ is strongly 2-absorbing. If $I J=0$, then applying (a) we again get that $X I=0$ or $X J=0$ and we are done.

(c) Suppose that $I J=J K=I K=0$. Set $I_{1}=I \cup J, I_{2}=J \cup K, I_{3}=I \cup K$. Clearly $I_{1} I_{2} I_{3}=0$ but since $I, J, K$ are not contained in $B, I_{i} I_{j} \neq 0$ for all $1 \leq i, j \leq 3$, a contradiction.

Conversely, suppose that $I_{1}, I_{2}, I_{3}$ are ideals of $S$ such that $I_{1} I_{2} I_{3}=0$. Set $I=$ $I_{1} I_{2}, J=I_{2} I_{3}, K=I_{1} I_{3}$. Then $I J=J K=I K=0$. Hence by (c) one of $I, J, K$, say $I$, is contained in $B$. Now by (a), $I_{1} I_{2}=0$ or we can assume that, say, $I_{1} \subseteq B$. However if $I_{1} \subseteq B$, then by (b), $I_{1} I_{2}=0$ or $I_{1} I_{3}=0$. These show that $S$ is strongly 2-absorbing. The result follows.

The following result is a semigroup counterpart of Theorem 2.4 in [2] describing the structure of 2-absorbing rings.

Theorem 2.5 $S$ is strongly 2-absorbing if and only if

(1) for every subset $X$ of $B$, ann $(X)$ is a prime ideal of $S$;

(2) one of the following conditions holds

(a) $B$ is a prime ideal of $S$

(b) $S$ contains prime ideals $P_{1}, P_{2}$ such that $P_{1} P_{2}=0$.

Proof Suppose that $S$ is strongly 2-absorbing. The condition (1) is precisely the condition (b) in Theorem 2.4. Suppose that $B$ is not a prime ideal. Then there are $s, t \in S \backslash B$ such that $s t \in B$. By Theorem 2.4(a), st $=0$ and $s B=t B=0$. Set $P_{1}=\operatorname{ann}(s), P_{2}=\operatorname{ann}(t)$. Then $B \subseteq P_{1}, B \subseteq P_{2}$ and $t \in P_{1}, s \in P_{2}$. Let $I, J$ be ideals of $S$ not contained in $P_{1}$. Then $s I \neq 0, s J \neq 0$, so by Theorem 2.4(a), $s I \nsubseteq B$, $s J \nsubseteq B$. Now $s I, s J,(t)$ are ideals of $S$ not contained in $B$ and $(t) s I=0,(t) s J=0$, so by Theorem 2.4(c), sIsJ $\neq 0$. Consequently $s I J \neq 0$ and $I J \nsubseteq P_{1}$, which shows that $P_{1}$ is a prime ideal of $S$. Similarly $P_{2}$ is a prime ideal of $S$. Note that $0 \neq t^{2} \in P_{1}(s, t), 0 \neq s^{2} \in P_{2}(s, t)$ but $P_{1} P_{2}(s, t)=0$. Consequently $P_{1} P_{2}=0$. These show the "only if" part. 
Conversely, suppose that $S$ satisfies the listed conditions. Take ideals $I_{1}, I_{2}, I_{3}$ of $S$ such that $I_{1} I_{2} I_{3}=0$. We have to show that $I_{i} I_{j}=0$ for some $1 \leq j \neq i \leq 3$. If $B$ is a prime ideal of $S$, then $I_{i} \subseteq B$ for some $1 \leq i \leq 3$ and we are done by (1). If $B$ is not a prime ideal, then applying (2) we get that there are $1 \leq i, j \leq 3$ such that $I_{i} \subseteq P_{1}, I_{j} \subseteq P_{2}$ and $I_{i} I_{j}=0$. If $i \neq j$ we are done. Thus suppose $i=j$ and, say, they are equal 1 . Then $I_{1} \subseteq B$ and $I_{2} I_{3} \subseteq \operatorname{ann}\left(I_{1}\right)$. However then from (1) it follows that $I_{1} I_{2}=0$ or $I_{1} I_{3}=0$ and we are done again.

Remark 2.6 One easily gets that if $P_{1}, P_{2}$ are prime ideals of an arbitrary (even non-commutative) semigroup $S$ such that $P_{1} P_{2}=0$, then $P_{1}, P_{2}$ are unique minimal prime ideals of $S$.

\section{Applications to rings}

In what follows $R$ is a commutative ring and $S(R)$ denotes the multiplicative semigroup of $R$.

We start with a simple lemma, which however plays a key role in the proof of Theorem 3.4 showing that $S(R)$ is 2-absorbing if and only if it is strongly 2-absorbing.

Lemma 3.1 Suppose that $A$ is a subsemigroup of the additive group of $R$ and $X, Y$ are subsets of $R$ such that for each $a \in A, a X=0$ or $a Y=0$. Then $A X=0$ or $A Y=0$.

Proof Suppose that there are $a, b \in A$ such that $a X \neq 0$ and $b Y \neq 0$. Then by the assumption $a Y=0$ and $b X=0$. Moreover $(a+b) X=0$ or $(a+b) Y=0$. In the former case $0=(a+b) X=a X \neq 0$ and in the latter $0=(a+b) Y=b Y \neq 0$. This contradiction proves the result.

Remark 3.2 This proof applies also to the case when $R$ is a semiring. One can check that in fact all the results below hold with the same proofs for semirings.

Corollary 3.3 If $A$ is a subsemigroup of the additive group of $R$ and ann(a) is $a$ prime ideal of $R$ for every $a \in A$, then $\operatorname{ann}(A)$ is a prime ideal of $R$.

Proof Let $x, y \in R$ and $A x y=0$. Since for every $a \in A$, $\operatorname{ann}(a)$ is a prime ideal, $a x=0$ or $a y=0$. Hence from Lemma 3.1 it follows that $A x=0$ or $A y=0$, so $\operatorname{ann}(A)$ is a prime ideal.

Theorem 3.4 The following conditions are equivalent

(a) $R$ is 2-absorbing;

(b) $S(R)$ is 2-absorbing;

(c) $S(R)$ is strongly 2-absorbing;

(d) for arbitrary ideals $I_{1}, I_{2}, I_{3}$ of $R$ satisfying $I_{1} I_{2} I_{3}=0$ there are $1 \leq i \neq j \leq 3$ such that $I_{i} I_{j}=0$. 
Proof It is evident that (a) and (b) are equivalent, (c) implies (b) and (d) implies (a).

Every ideal of $R$ is obviously an ideal of $S(R)$. For a given ideal $I$ of $S(R)$ denote by $\bar{I}$ the ideal of $R$ generated by $I$. It is clear that $\overline{I J}=\bar{I} \bar{J}$ (where $\bar{I} \bar{J}$ denotes the product of ring ideals). These easily show that (c) and (d) are equivalent.

It remains to show that (a) implies (c). For that in view of Proposition 2.3 we have to show that if $F_{1}, F_{2}, F_{3}$ are ideals of $S(R)$ generated by $\leq 2$ elements each and $F_{1} F_{2} F_{3}=0$, then $F_{i} F_{j}=0$ for some $1 \leq i \neq j \leq 3$. Let $n$ be the sum of the minimal numbers of generators of $F_{i}$. Obviously $3 \leq n \leq 6$. Since $S(R)$ is 2 -absorbing the result holds for $n=3$, i.e., when each $F_{i}$ is a principal ideal of $S(R)$. Thus assume that $n \geq 4$, say, $F_{1}=(s, t)$ and the result holds if the sum of the numbers of generators is $\leq n-1$. If $F_{2} F_{3}=0$, we are done. Otherwise, since for each $f \in \bar{F}_{1},(f) F_{2} F_{3}=0$, our assumptions on $n$ implies that $f F_{2}=0$ or $f F_{3}=0$. Now applying Lemma 3.1 we get that $F_{1} F_{2}=0$ or $F_{1} F_{3}=0$.

Note that for every subset $X$ of $R$ the annihilators of $X$ in $R$ and in $S(R)$ coincide. Moreover if $I$ is an ideal of $R$, then $I$ is a prime ideal of $R$ if and only if it is a prime ideal of $S(R)$. It is also clear that the prime radical of $R$ coincides with the prime radical of $S(R)$. These, Theorem 2.5, Corollary 3.3 and Theorem 3.4 give the following

Theorem 3.5 ([2], Theorem) $R$ is 2-absorbing if and only if for every $x \in B, \operatorname{ann}(x)$ is a prime ideal of $R$ and one of the following conditions holds

(a) $B$ is a prime ideal of $R$ or

(b) $R$ contains prime ideals $P_{1}, P_{2}$ such that $P_{1} P_{2}=0$.

Let $G$ be an abelian group and let $R$ be a $G$-graded ring. Recall that $R=$ $\bigoplus_{g \in G} R_{g}$, the direct sum of additive subgroups $R_{g}$ of $R$, with $R_{g} R_{h} \subseteq R_{g h}$ for all $g, h \in G$. Elements of $\bigcup_{g \in G} R_{g}$ are called homogeneous. Every $r \in R$ can be written as a finite sum $r=\sum_{g} r_{g}$, where $r_{g} \in R_{g}$ is called the homogeneous component of $r$. The set $\operatorname{supp}(r)=\left\{g \in G \mid r_{g} \neq 0\right\}$ is called the support of $r$. An ideal $I$ of $R$ is called homogeneous if $I=\bigoplus_{g \in G}\left(I \cap R_{g}\right)$.

For more details concerning graded rings we refer to [4].

Denote by $S_{h}(R)$ the multiplicative semigroup of homogeneous elements of $R$. For a given ideal $I$ of $S_{h}(R)$ denote by $\bar{I}$ the ideal of $R$ generated by $I$. It is clear that $\bar{I}$ is a homogeneous ideal of $R$ and $\overline{I J}=\bar{I} \bar{J}$. For every homogeneous ideal $I$ of $R$ the set $I^{*}$ of homogeneous components of elements from $I$ is an ideal of $S_{h}(R)$ and $I$ is a graded prime ideal of $R$ if and only if $I^{*}$ is a prime ideal of $S_{h}(R)$ as well as the prime radical $B_{h}(R)$ of $S_{h}(R)$ coincides with $B_{h}^{*}$, where $B_{h}$ is the graded prime radical of $R$. Finally for every subset $X$ of $R$ consisting of homogeneous elements $\operatorname{ann}(X)$ is a homogeneous ideal of $R$. These and Theorem 2.5 give

Theorem 3.6 For a given $G$-graded ring $R$ the following conditions are equivalent

(a) $S_{h}(R)$ is strongly 2-absorbing;

(b) For arbitrary homogeneous ideals $I_{1}, I_{2}, I_{3}$ satisfying $I_{1} I_{2} I_{3}=0$ there are $1 \leq$ $i \neq j \leq 3$ such that $I_{i} I_{j}=0$; 
(c) For every subset $X$ of $B$ consisting of homogeneous elements, ann $(X)$ is a graded prime ideal and one of the following conditions holds

- $B_{h}$ is a graded prime ideal of $R$ or

- $R$ contains graded prime ideals $P_{1}, P_{2}$ such that $P_{1} P_{2}=0$.

If $R$ is a $G$-graded 2-absorbing ring then by Theorem 3.4, $S_{h}(R)$ is strongly 2absorbing. The converse does not hold. For instance if $G$ is a finite abelian group of order $\geq 3$ and $F$ is an algebraically closed field of characteristic 0 and $R$ is the group algebra of $G$ over $F$, then obviously $S_{h}(R)$ is strongly 2 -absorbing but, since $R$ is isomorphic to the direct sum of $|G|$ copies of $F, R$ is not 2-absorbing. The following result, which generalizes Theorem 4.15 in [1], shows that the converse holds if $G$ is torsion-free.

Theorem 3.7 Suppose that $R$ is a $G$-graded ring and $G$ is torsion-free. If $S_{h}(R)$ is strongly 2-absorbing, then $R$ is 2-absorbing (or, equivalently, $S(R)$ is 2-absorbing).

Proof It is well known and easy to check that, since $G$ is torsion-free, a homogeneous ideal of $R$ is prime if and only if it is graded prime as well as $B=B_{h}$. Consequently if $B_{h}$ is a graded prime ideal of $R$, then $B$ is a prime ideal of $R$. If $B$ is not a prime ideal, then by Theorems 3.6(c), $R$ contains graded prime ideals $P_{1}, P_{2}$ such that $P_{1} P_{2}=0$. Hence $P_{1}, P_{2}$ are also prime ideals of $R$. Hence in view of Theorem 3.5 to get the result it suffices to show that for each $0 \neq x \in B, \operatorname{ann}(x)$ is a prime ideal of $R$. Suppose that for some $a, b \in R \backslash a n n(x), a b \in \operatorname{ann}(x)$, i.e., $x a b=0$ but $x a \neq 0$ and $x b \neq 0$. Choose them in such a way that $n=\operatorname{card}(\operatorname{supp}(x))+\operatorname{card}(\operatorname{supp}(a))+\operatorname{card}(\operatorname{supp}(b))$ is minimal. Moreover, we can assume that $G$ is generated by the union of the supports of $x, a, b$. Then $G$, being a finitely generated abelian torsion-free group, is linearly ordered. Obviously $n \geq 3$ and by Theorem 3.6(c) not all $x, a, b$ are homogeneous, so $n>3$. Let $g, h, t$ be the largest elements in $\operatorname{supp}(x), \operatorname{supp}(a), \operatorname{supp}(b)$, respectively. Then $x_{g} a_{h} b_{t}=0$ and by Theorem 3.6(c) we can assume that $x_{g} a_{h}=0$. If $x a_{h}=0$, then $a_{h} \neq a$ and $x\left(a-a_{h}\right) b=0$ and $\operatorname{card}(x)+\operatorname{card}\left(a-a_{h}\right)+\operatorname{card}(b)<n$, so since $x b \neq 0$, we get $x\left(a-a_{h}\right)=0$. These give $x a=0$, a contradiction. Thus $x a_{h} \neq 0$. Now $\left(x a_{h}\right) a b=0$ and $\operatorname{card}\left(\operatorname{supp}\left(x a_{h}\right)\right)+\operatorname{card}(\operatorname{supp}(a))+\operatorname{card}(\operatorname{supp}(b))<n$, so $x a_{h} a=0$ or $x a_{h} b=0$. If the former holds and $x$ is a homogeneous element, then for each homogeneous component $a_{t}$ of $a$ we have $x a_{h} a_{t}=0$. Since $a$ is not homogeneous, $x a_{h} \neq 0$ and $\operatorname{card}(\operatorname{supp}(x))+2<n$, we get that $x a_{t}=0$, a contradiction. In the latter case $\operatorname{card}(\operatorname{supp}(x))+\operatorname{card}\left(\operatorname{supp}\left(a_{h}\right)\right)+\operatorname{card}(\operatorname{supp}(b))<n$ and $x a_{h} \neq 0$ as well as $x b \neq 0$, so we get a contradiction again. This finishes the proof.

The following example shows that in Theorem 3.7 it is not enough to assume merely that $S_{h}(R)$ is 2-absorbing.

Example 3.8 Let $F$ be a field and $A=F[x, y] / I$, where $I$ is the ideal of $F[x, y]$ generated by $x^{2}, y^{2}$. Set $a=x+I$ and $b=y+I$. The $F$-subalgebra $R=F+$ $F a z+F b z^{2}+F a b z^{3}$ of $A[z]$ is graded in a canonical way by the additive group of integers. Note that for $t=a z+b z^{2}$ we have $t^{3}=0$ and $t^{2} \neq 0$, so $R$ is not 2absorbing. However every non-invertible element of $S_{h}(R)$ is square-zero, so $S_{h}(R)$ is 2-absorbing. 


\section{On $n$-absorbing and strongly $n$-absorbing rings}

A finite number of ideals of $R$ (some ideals can appear several times) will be called a collection of ideals if their product is equal 0 .

Let $n$ be an integer $\geq 2$. A ring $R$ is called strongly $n$-absorbing (c.f. [1]) if the following condition is satisfied:

(*) every collection of $n+1$ ideals of $R$ contains a collection of $n$ ideals.

If the condition (*) is satisfied for collections of $n+1$ principal ideals, then $R$ is called $n$-absorbing.

It is evident that strongly $n$-absorbing rings are $n$-absorbing. In [1] it was conjectured that the converse holds as well. We will show that it is indeed the case if the additive group of $R$ is torsion-free.

Arguments similar to those applied in the proof of Proposition 2.3 give the following characterization of strongly $n$-absorbing rings.

Proposition 4.1 A ring $R$ is strongly $n$-absorbing if and only if it satisfies the condition (*) for ideals generated by $\leq n$ generators each.

Proof If $R$ is not strongly $n$-absorbing, then there exists a collection $I_{1}, \ldots, I_{n+1}$ of ideals of $R$ such that no $n$ of these ideals is a collection. Hence for every $1 \leq i \leq n+1$ there are $f_{i j} \in I_{j}, 1 \leq j \neq i \leq n+1$ whose product is not equal 0 . Let, for each $1 \leq j \leq n+1, F_{j}$ be the ideal of $R$ generated by the set of all $f_{i j}$. It is clear that $F_{1}, F_{2}, \ldots, F_{n+1}$ is a collection of ideals but no $n$ of these ideals form a collection. This shows the "if" part. The "only if" part is evident.

Theorem 4.2 If $R$ is $n$-absorbing and the additive group of $R$ is torsion-free, then $R$ is strongly $n$-absorbing.

Proof In view of Proposition 4.1 it suffices to show that if $F_{1}, F_{2}, \ldots, F_{n+1}$ is a collection of ideals of $R$ and each of them is generated by $\leq n$ elements, then for some $1 \leq j \leq n+1$ the product of all $F_{i}$ for $i \neq j$ is equal 0 . Define the index of a collection of finitely generated ideals as the sum of minimal numbers of their generators. It is clear that if $s$ is the index of the collection $F_{1}, \ldots, F_{n+1}$, then $n+1 \leq s \leq n(n+1)$. If $s=n+1$, then all ideals $F_{i}$ are principal, so the claim holds as $R$ is $n$-absorbing. Thus suppose that $s>n+1$ and the result holds for collections of $n+1$ ideals with index smaller than $s$. Then at least one of the $F_{i}$, say $F_{1}$, is not principal. If the result does not hold for $s$, then $F_{2} F_{3} \cdots F_{n+1} \neq 0$. For each $f \in F_{1}, f F_{2} F_{3} \cdots F_{n+1}=0$ and since $I_{1}$ is not principal, the index of the collection $(f), F_{2}, \ldots, F_{n+1}$ is $<s$. Thus for some $2 \leq k \leq n+1$, we have $f T_{k}=0$, where $T_{k}$ is the product of all ideals $F_{2}, \ldots, F_{n+1}$ except $F_{k}$. Consequently $F_{1} \subseteq \operatorname{ann}\left(T_{2}\right) \cup \operatorname{ann}\left(T_{3}\right) \cup \cdots \cup \operatorname{ann}\left(T_{n+1}\right)$. Obviously $F_{1}$ and all $\operatorname{ann}\left(T_{i}\right)$ are subgroups of the additive group of $R$. It is well known (cf. [3]) that these imply that for a positive integer $m, m F_{1}$ is contained in $\operatorname{ann}\left(T_{i}\right)$ for some $2 \leq i \leq n+1$. Hence $m F_{1} T_{i}=m \bar{F}_{i}=0$, where $\bar{F}_{i}$ is the product of all $F_{1}, \ldots, F_{n+1}$ except $F_{i}$. However $\bar{F}_{i} \neq 0$ and the additive group of $R$ is torsionfree, so we get a contradiction. 
Open Access This article is distributed under the terms of the Creative Commons Attribution License which permits any use, distribution, and reproduction in any medium, provided the original author(s) and the source are credited.

\section{References}

1. Anderson, D.F., Badawi, A.: On n-absorbing ideals of commutative rings. Commun. Algebra 39, 16461672 (2011)

2. Badawi, A.: On 2-absorbing ideals of commutative rings. Bull. Aust. Math. Soc. 75, 417-429 (2007)

3. McCoy, N.H.: A note on finite unions of ideals and subgroups. Proc. Am. Math. Soc. 8, 633-637 (1957)

4. Năstǎsescu, C., Van Oystaeyen, F.: Graded Ring Theory. North-Holland, Amsterdam (1982) 